\title{
correspondence
}

\section{Swine influenza}

SIR,-The recent description of a human outbreak of influenza virus in an American military camp has justifiably given rise to a great deal of concern. The possibility that the virus involved might cause a severe pandemic must be seriously considered and it is perfectly understandable that vigorous measures be taken to deal with this contingency. Of such measures, the most effective is vaccination with the swine virus and the technology and resources at present available should make possible the production of sufficient quantities of inactivated vaccine for extensive use before the onset of a possible pandemic is anticipated.

Use of such a vaccine on a large scale, however, poses several problems apart from the cost of vaccine production and administration. In the first place, it may be difficult to justify the use of this vaccine for the protection of high risk subjects for whom vaccination is usually recommended, as the majority of such subjects belong to age groups in which antibody (and presumably resistance) to the swine virus is found in a high proportion of individuals due to exposure during 1918 and subsequent years of prevalence of this virus in human populations. Vaccination of younger age groups would be more justified especially considering the possibility of high mortality among young subjects as in the 1918 pandemic. It is difficult, not to say impossible, to estimate the probability of epidemic or pandemic spread of the swine virus.

This uncertainty should not dampen our efforts towards control, but what is to be our future action in the not too unlikely event of epidemics not being observed in the coming season? The risk will still be there in succeeding years and to ignore it will be unwise. If presently feasible inactivated virus vaccine is to be relied upon, the relatively short-lived protection it confers would lead to the daunting prospect of repeated early vaccination against a virus which might not appear at all. A more sensible solution would be to stockpile a strategic reserve of vaccine. Surveillance programmes in operation at present should be more than adequate to detect early signs of epidemic activity of the virus in time to enable the effective distribution and administration of vaccine. Adoption of this policy would make economic sense as the initial investment would be compensated by savings in avoiding the unnecessary use of vaccine in nonepidemic years.

If a strategic reserve of vaccine is acceptable as a potential defence against foot-and-mouth disease of cattle, this would be preferable to the possibly superfluous use of vaccine on the massive scale anticipated in the USA or to the vaccination of already resistant subjects as proposed in this country.

\section{Animal Virus Research Institute, Pirbright, Surrey, UK}

\section{Alternative refrigerants}

SIR,-F. A. Cotton's letter (Correspondence, March 25, page 280) is an oversimplification although not, perhaps, a serious one. The fluorocarbon manufacturers estimate that the mean life of a fluorocarbon between manufacture and release to the atmosphere is about six months for an aerosol propellant and about two years for a refrigerant. The latter statement is surprising but is perhaps explained by the fact that a proportion of refrigerants is used in freeze drying and released immediately to the atmosphere, and also that in North America, at any rate, a considerable quantity is used in the air conditioning systems of cars which, perhaps sooner rather than later, are involved in head to tail collisions and release their fluorocarbons as a result.

Birks and Leck (Correspondence March 4, page 8) omitted to state that already in North America about 50\% of total refrigerant used is refrigerant 22 which is unsuitable as an aerosol propellant. This contains a carbonhydrogen bond and, for reasons not completely understood but probably because this bond is destroyed by $\mathrm{OH}$ radicals which it can encounter in the lower atmosphere, its lifetime in the atmosphere is rather short, probably around three years, as compared with the 80-100 year life of refrigerant 12 . It thus appears that refrigerant 22 is unlikely to cause a problem as far as stratospheric ozone is concerned.

The reason commonly given by the chemical manufacturers for not using refrigerant 22 exclusively is that this would leave manufacturing capacity unused as it is not possible to manufacture R-22 in a plant designed for $\mathrm{R}-12$. However, I have been informed by a particular manufacturer that he routinely switches his plants from $\mathrm{R}-22$ to R-12 according to demand. I was not previously aware that an $\mathrm{R}-22$ refrigerant plant was much more expensive but, if this is so, it is difficult to understand why already half the refrigerant used is R-22.

There thus seems to be no valid reason why we should not dispense with the expensive, trivial and possibly dangerous convenience of the aerosol spray can-remembering that fluorocarbons are used almost exclusively in "personal care" products such as hair sprays and deodorants - and still retain the benefits of refrigeration.

\section{York University, Ontario}

\section{Human anatomy}

SiR-Kenneth Mellanby (February 19, page 523) remarked that model was a term capable of abuse, and that in Soho the word probably would not lead to useful scientific research. A week or two earlier the asymmetry of the scrotal sac of male homo sapiens had been discussed using artistic models as criteria.

Both these publications reveal the curious reluctance of scientists to research into human anatomy and relate it to function, disease and sociology. Such studies might usefully be termed gross morphological enquiries, in contrast with the vague constitutional studies which had a short flowering in the late nineteenth century and midtwentieth century.

I have found, for instance, that asymmetry is commonplace in the human physique. The circumference ratios (right/left) of thirty right-handed women in the 18-22 years age group were measured, and averaged 1.02 (ankle), 1.06 (calf), 1.05 (thigh), 1.09 (biceps). The volume of the mammary glands also differs, although supportive garments do not allow for this fact; the result is that the right mammary gland, if supported well, implies a looser support for the left. The explanation of these asymmetries is presumably in the greater use of the muscles underlying the right hand limbs.

No evidence for this asymmetry can be found in the paintings of Titian or Rubens in the National Gallery, London or in Rodin's The Kiss.

Anthony B. HaRris

Department of Food Sciences, The Polytechnic of North London, London N7, UK 